\title{
Neural Progenitor Cells Generate Motoneuron-Like Cells to Form Functional Connections With Target Muscles After Transplantation Into the Musculocutaneous Nerve
}

\author{
Huanxing Su, ${ }^{1,2}$ Wenming Zhang, $\dagger^{1}$ Xiaoying Yang, ${ }^{* 1}$ Dajiang Qin,* Yanhua Sang,* \\ Chaoyang Wu, $\dagger$ Wai-Man Wong, ${ }^{*}$ Qiuju Yuan,* Kwok-Fai So, $* \ddagger \S$ and Wutian Wu* $* \S \mathbb{I}$ \\ *Department of Anatomy, The University of Hong Kong, Pokfulam, Hong Kong SAR, China \\ $\dagger$ Department of Orthopaedics, The First Affiliated Hospital of Fujian Medical University, Fujian, China \\ $\ddagger$ State Key Laboratory of Brain and Cognitive Sciences, Li Ka Shing Faculty of Medicine, \\ The University of Hong Kong, Pokfulam, Hong Kong SAR, China \\ $\S$ Joint Laboratory for Brain Function and Health (BFAH), Jinan University \\ and The University of Hong Kong, Jinan University, Guangzhou, China \\ IfResearch Center of Reproduction, Development and Growth, Li Ka Shing Faculty of Medicine, \\ The University of Hong Kong, Pokfulam, Hong Kong SAR, China
}

\begin{abstract}
Neural progenitor cells (NPCs) are suggested to be a valuable source of cell transplant in treatment of various neurological diseases because of their distinct attributes. They can be expanded and induced to differentiate in vitro. However, it remains uncertain whether in vitro expanded NPCs have the capacity to give rise to functional motoneurons after transplantation in vivo. Here, we showed that in vitro expanded NPCs, when transplanted into the musculocutaneous nerve, generated motoneuron-like cells that exhibited typical morphology with large cell bodies, expressed specific molecules, and extended axons to form functional connections with the target muscle. In contrast, transplanted NPCs failed to yield motoneurons in the injured ventral horn of the spinal cord. The results of the study demonstrate that NPCs have the potential to generate functional motoneurons in an appropriate environment. The distinct differentiating fate of NPCs in the musculocutaneous nerve and the injured ventral horn suggests the importance and necessity of modifying the host microenvironment in use of NPCs for cell replacement therapies for motoneuron diseases.
\end{abstract}

Key words: Neural progenitor cells (NPCs); Motoneurons; Nerve injury; Spinal cord; Transplantation

\section{INTRODUCTION}

Since the success in isolating and expanding neural progenitor cells (NPCs) from specific regions of the developing and adult CNS $(19,22,24,25,34)$, NPCs have been widely used in neurological disorder repair. Compared to other stem cells, NPCs are already committed to a neural fate and hence will be easier to differentiate into mature neural phenotypes (1). They are expected to replace lost, damaged, or dysfunctional neurons in cell-based therapies for the treatment of a variety of neurological diseases such as Parkinson's disease, Alzheimer's disease, Huntington's disease, amyotrophic lateral sclerosis (ALS), etc. However, many studies have reported that grafted NPCs differentiate predominantly into glial phenotypes and show very limited ability to produce region-specific neuronal phenotypes in the adult CNS $(3,8,11,28,35)$.

The unfavorable environment of adult CNS is considered one of the factors responsible for the failure of grafted NPCs to generate region-specific neurons. An alternative explanation, however, is that in vitro expanded NPCs may lose the ability to respond to cues for regional specification $(14,31)$. They are supposed to have limited plasticity and unable to give rise to region-specific neurons. Motoneuron diseases such as ALS, spinal bulbar muscular atrophy (SBMA), spinal muscular atrophy (SMA), and spinal muscular atrophy with respiratory distress 1 (SMARD1) are characterized

Received July 15, 2011; final acceptance February 8, 2012. Online prepub date: September 7, 2012.

${ }^{1}$ These authors provided equal contribution to this work.

${ }^{2}$ Present address: State Key Laboratory of Quality Research in Chinese Medicine and Institute of Chinese Medical Sciences, University of Macau, Macao SAR, China.

Address correspondence to Wutian Wu, Department of Anatomy, Li Ka Shing Faculty of Medicine, The University of Hong Kong, 21 Sassoon Road, Hong Kong SAR, China. Tel: +852-2819-9187; Fax: +852-2817-0857; E-mail: wtwu@ hkucc.hku.hk 
by degeneration of motoneurons, with resulting muscle wasting and paralysis. The selective degeneration of motoneurons is encouraging for cell replacement strategies using stem cell-derived motoneurons (13). The usefulness of NPCs would be vastly limited for replacement strategies for the treatment of motoneuron diseases if they are inherently unable to generate the projecting cells, although they are easy to be manipulated and propagated in large quantities.

In the present study, we transplanted spinal cordderived NPCs into the injured ventral horn and the transected musculocutaneous nerve to investigate whether they could yield motoneurons after transplantation in vivo. Our study showed that NPCs, when transplanted into the musculocutaneous nerve, produced motoneuronlike cells that exhibited typical morphology with large cell bodies, expressed specific molecules, and formed functional connections with the target muscle. In contrast, NPCs failed to generate motoneurons in the injured ventral horn of the spinal cord. Our results provide evidence that NPCs have the potential to efficiently generate functional motoneurons in an appropriate environment, and the failure of motoneuron generation in the injured ventral horn suggests the importance and necessity of modifying the host microenvironment in use of NPCs for cell replacement therapies for motoneuron diseases.

\section{MATERIALS AND METHODS}

All surgical interventions and subsequent care and treatment were approved by the Committee on the Use of Live Animals for Teaching and Research of the University of Hong Kong.

\section{Cell Isolation, Culture, and Characterization}

Under sterile conditions, spinal cords from embryonic day 13.5 transgenic Sprague-Dawley rats expressing green fluorescent protein (GFP; "green rat" CZ-004; SLC, Shizuoka, Japan) were dissected out and prepared for stem cell culture following procedures described previously (27). Briefly, the spinal cord was separated from surrounding connective tissue. After peeling off the meninges, the cord was transferred into a $15-\mathrm{ml}$ centrifuge tube containing stem cell culture medium (described below) and dissociated to a single-cell suspension by gentle mechanical trituration through a fire-polished Pasteur pipette. The dissociated cells were filtered through a cell strainer (BD Falcon, San Jose, CA, USA) and plated onto dishes coated with poly-L-lysine (PLL) $(13.3 \mu \mathrm{g} /$ ml; Sigma, St. Louis, MO, USA) and laminin $(20 \mu \mathrm{g} / \mathrm{ml}$; Sigma). Stem cell culture medium consisted of DMEMF12 (Gibco, Grand Island, NY, USA), bovine serum albumin (BSA) (1 mg/ml; Sigma), B27 (20 $\mu \mathrm{l} / \mathrm{ml}$; Invitrogen, Carlsbad, CA, USA), N2 (10 $\mu \mathrm{l} / \mathrm{ml}$; Invitrogen), basic fibroblast growth factor (bFGF) $(20 \mathrm{ng} / \mathrm{ml}$; Peprotech,
Rocky Hill, NJ, USA), and Pen-Strep (100 IU/ml; Invitrogen). Cells were maintained in an incubator with a humidified atmosphere containing $5 \% \mathrm{CO}_{2}$ at $37^{\circ} \mathrm{C}$. The medium was changed every 2 days. At around $80 \%$ confluence, cells were passaged at a ratio of 1:4 to 1:6 after initial plating. These subcultured cells were designated as "first passage" (P1). All experiments were carried out using P1 NPCs in this study.

For in vitro experiments, cultured cells on coverslips were fixed in $4 \%$ paraformaldehyde dissolved in $0.1 \mathrm{M}$ phosphate buffer (PB) for $20 \mathrm{~min}$. After several washes with $0.01 \mathrm{M}$ PBS, cells were processed for immunocytochemistry. The following primary antibodies were used to stain the cells: mouse anti-nestin antibody (1:200; Chemicon) for characterization of undifferentiated NPCs; mouse anti-neuronal nuclei (NeuN) (1:200; Chemicon), mouse anti-neurofilament 200 (NF200) (1:500; Chemicon), rabbit anti-choline acetyltransferase (ChAT) (1:200; Chemicon), and mouse anti-MAP2 (1:400; Chemicon) for neurons; and rabbit anti-glial fibrillary acidic protein (GFAP) antibody (1:1,000; Sigma) and mouse anti-Rip antibody (1:50; a generous gift from Dr. X. M. Xu, University of Louisville, Kentucky, USA) for astrocytes and oliogodendrocytes, respectively. Cell slides were then counterstained with DAPI to stain nuclei and coverslipped with antifade mounting medium (FluorSave, Calbiochem). The images were taken with a laser confocal microscope (LSM510 META, Carl Zeiss Meditec, Jena, Germany).

\section{Animal Surgery and Cell Transplantation}

Thirty-two adult female Sprague-Dawley rats (220$250 \mathrm{~g}$ ) were anesthetized with an intraperitoneal injection of ketamine $(80 \mathrm{mg} / \mathrm{kg})$ and xylazine $(8 \mathrm{mg} / \mathrm{kg})$. The animals were divided into two groups. The animals in the first group $(n=16)$ were subdivided evenly into two groups: one group received cell injection into both the root avulsion-injured ventral horn of the spinal cord and the transected musculocutaneous nerve, and the other group received cell injection into the root avulsion-injured ventral horn only. Root avulsion was performed as described previously (29). Briefly, the rat was placed in a supine position, and the right seventh cervical (C7) spinal nerve of the brachial plexus was identified and isolated. Extravertebral avulsion was carried out by pulling the C7 spinal nerve out with a pair of microhemostatic forceps. The avulsed ventral and dorsal roots together with the dorsal root ganglia were cut away from the distal stump of the C7 spinal nerve and examined under a microscope to confirm the success of the surgery. After root avulsion, the animals had the right musculocutaneous nerve isolated and then transected at the proximal site. One microliter of cell suspension was slowly injected into 
the musculocutaneous nerve with a Hamilton syringe (needle gauge 30) with a sharpened tip. Immediately after cell transplantation into the musculocutaneous nerve, the same animal then received cell graft into the avulsed ventral horn. The dorsal neck musculature was incised, and a laminectomy was performed at C6. Under an operating microscope, a glass micropipette $(100-\mu \mathrm{m}$ outer diameter) connected to a Hamilton syringe was stereotactically advanced into the avulsed ventral horn (ML: $-0.8 \mathrm{~mm}$ and DV: $-1.5 \mathrm{~mm}$ from dura) at the right C7 spinal segment. One microliter of cell suspension was slowly injected over $5 \mathrm{~min}$ into the avulsed ventral horn. P1 NPCs derived from embryonic day 13.5 GFP rat spinal cords were dissociated and resuspended in NB medium at a concentration of $1.0 \times 10^{5}$ cells $/ \mu 1$ and placed on ice for the duration of the grafting session. Cell viability was assessed with trypan blue at the end of transplantation, and typically more than $90 \%$ of the cells excluded the dye. In the second group, the animals were subdivided evenly into two groups: receiving either cell transplantation $(n=8)$ or vehicle injection $(n=8)$ into the transected musculocutaneous nerve. The dura was closed with 10-0 sutures, while muscle fascia were reapposed and sutured with 4-0 sutures, and the skin was closed with wound clips. In the control group, animals received an injection of the same volume of NB medium instead of cell suspension into the musculocutaneous nerve and the avulsed ventral horn. The animals were kept warm and allowed to recover from anesthesia before being returned to their cages. All the animals were allowed to survive for 12 weeks.

\section{Tissue Processing}

At the end of the survival period, eight animals in the first group were killed with a lethal dose of sodium pentobarbital and perfused intracardially with $0.01 \mathrm{M}$ PBS (pH 7.4), followed by $200-300 \mathrm{ml}$ of fixative solution containing $4 \%$ paraformaldehyde in $0.1 \mathrm{M} \mathrm{PB} \mathrm{(pH} \mathrm{7.4).}$ The right musculocutaneous nerve and the $\mathrm{C} 7$ segments of spinal cords were harvested and fixed in fresh fixative solution overnight and subsequently placed in $30 \%$ sucrose- $0.1 \mathrm{M} \mathrm{PB}$ at $4^{\circ} \mathrm{C}$ for 2-3 days. The $\mathrm{C} 7$ segments were then cut into $30-\mu \mathrm{m}$ sections on a cryostat. The serial sections were collected in $0.01 \mathrm{M} \mathrm{PBS}$ and kept at $4^{\circ} \mathrm{C}$ for further study.

\section{Immunohistochemistry}

Longitudinal nerve sections and cross spinal cord sections were collected for immunostaining. Sections were washed in $0.01 \mathrm{M}$ PBS, blocked in $10 \%$ goat serum for $1 \mathrm{~h}$, and incubated with primary antibodies at $4^{\circ} \mathrm{C}$ overnight. Neurons were detected with mouse anti-neuronal nuclei (NeuN) (1:200; Chemicon), mouse anti-neurofilament 200 (NF200) (1:500; Chemicon), and rabbit anti-choline acetyltransferase (ChAT) (1:200; Chemicon); astroglial cells were identified using rabbit anti-glial fibrillary acidic protein (GFAP) $(1: 1,000$; Sigma); myelinating cells were identified using mouse anti-adenomatus polyposis coli (APC) (1:200; BD Biosciences Pharmingen, San Jose, CA, USA), S-100 $\beta$ (1:200; BD Biosciences Pharmingen), and myelin basic protein (MBP) (1:500; BD Biosciences Pharmingen); rabbit anti-GFP (1:800; Abcam) was used to intensify the GFP signal. Sections were washed in 0.01 M PBS, and species-specific fluorescence-conjugated secondary antibodies (goat anti-mouse or anti-rabbit) conjugated to Alexa 568 (1:400; Molecular Probes) were applied for $2 \mathrm{~h}$ at $20^{\circ} \mathrm{C}$. After being washed in $0.01 \mathrm{M}$ PBS, sections were then counterstained with 4',6-diamidino2-phenylindole (DAPI) to stain nuclei and coverslipped with antifade mounting medium (FluorSave, Calbiochem, San Diego, CA, USA). To assess differentiation patterns of transplanted cells, we stained two tissue sections per rat (eight animals in each group) for each cell marker and then counted the number of GFPexpressing cells colocalized with the cell marker in 10 random fields per section at $40 \times$ magnification using a confocal microscope (LSM510 META, Carl Zeiss Meditec, Jena, Germany). On average, around 200 cells were counted per section. All quantification was performed in an unbiased stereological manner.

\section{Electromyographic Measurements}

At the end of the study, the animals in the second group were killed (eight animals from the cell implantation group and eight from the vehicle control group), and the musculocutaneous nerve and biceps brachii muscle were quickly removed and transferred into a recording chamber that was continuously perfused with room-temperature oxygenated Tyrode's solution $\left(125 \mathrm{mM} \mathrm{NaCl}, 24 \mathrm{mM} \mathrm{NaHCO}_{3}, 5.4 \mathrm{mM}\right.$ $\mathrm{KCl}, 1 \mathrm{mM} \mathrm{MgCl}, 1.8 \mathrm{mM} \mathrm{CaCl}_{2}$, and $5 \%$ dextrose) at a flow rate of $4-5 \mathrm{ml} / \mathrm{min}$. Electromyographic (EMG) recordings were obtained using a suction electrode on the surface of the muscle, while a second suction electrode was used to stimulate the nerve. EMG signals were amplified with a differential amplifier (EX4-400, Dagan), captured using Axoscope 9.2 software (Molecular Devices). To confirm that the muscle contractions resulted from neurotransmission by graft-derived motoneurons, the competitive nicotinic antagonist D-tubocurarine (Research Biochemicals International) was applied to the recording solution. EMG activity was recorded with D-tubocurarine and after a 5-h washout period.

\section{Neuromuscular Junction Labeling}

Wet weight of the biceps brachii muscle was measured and compared between the cell transplant and control groups. The musculocutaneous nerve and the biceps 


\section{NPCs in stem cell culture medium}

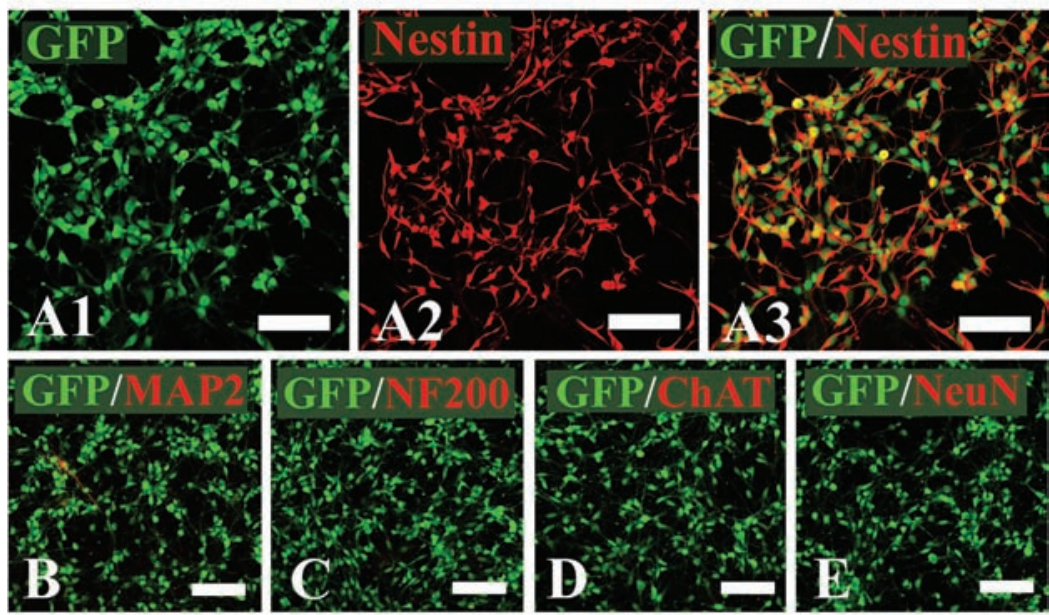

NPCs in differentiating medium
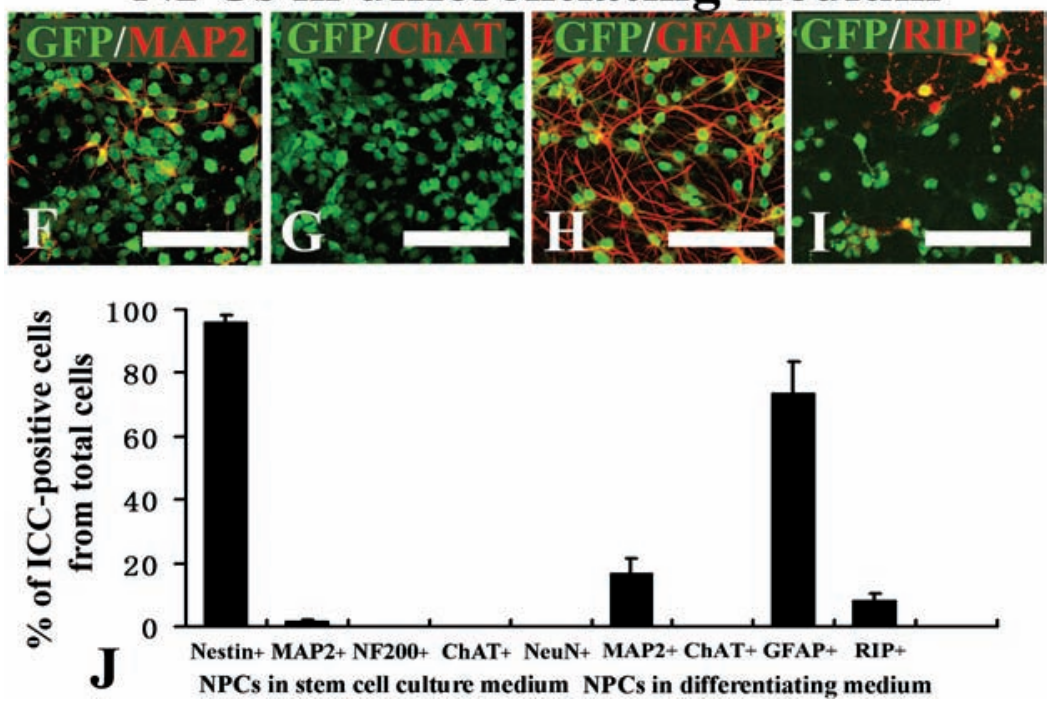

Figure 1. P1 neural progenitor cell (NPC) characterization and differentiation pattern in vitro. (A) P1 NPCs were plated as a monolayer on poly-L-lysine (PLL)/laminin-coated culture dishes in basic fibroblast growth factor (bFGF)-supplemented culture medium. The green fluorescent protein (GFP)-positive cells showed a bipolar or multipolar morphology with round-shaped cell bodies (A1). Immunocytochemistry of these cells revealed efficient expression of the progenitor cell marker nestin (A2). The majority of the GFP-expressing cells were nestin positive (A3). (B) Microtubule-associated protein 2 (MAP2) staining showed that less than 1\% cells in P1 NPC culture were positive $(0.96 \pm 0.1 \%)$. (C) No neurofilament 200 (NF200)-positive cells were found in the culture. (D) No choline acetyltransferase (ChAT)positive cells were found in the culture. (E) No neuronal nuclei (NeuN)-positive cells were found in the culture. (F-I) When bFGF was removed from the culture and replaced with $1 \%$ fetal bovine serum (FBS) for 5 days, a number of NPCs (16.7\%) differentiated into MAP2positive neurons (F). The majority of NPCs (73.5\%) differentiated into glial fibrillary acidic protein (GFAP)-positive astrocytes (H), and a small population of NPCs (8.4\%) into Rip-positive oligodendrocytes (I). However, no ChAT-positive cells were found in the culture (G). (J) Percentage of ICC-positive cells presented as mean \pm SEM (ICC, immunocytochemistry). Scale bars: $80 \mu \mathrm{m}$.

brachii muscle were then fixed in $3.7 \%$ formaldehyde and cryosectioned at $30 \mu \mathrm{m}$. The nerve sections were used for immunohistological and EM studies. Biceps brachii muscle slices were incubated with $1 \mu \mathrm{g} / \mathrm{ml}$ rhodamine-conjugated $\alpha$-bungarotoxin ( $\alpha$-BT; 1:400; Molecular Probes) for $1 \mathrm{~h}$ at room temperature to visualize acetylcholine receptors
(ACHRs). The muscle slices were then immunostained with NF200 to label nerve terminals.

\section{Immunoelectron Microscopy}

Cryostat nerve sections were processed for immunoelectron microscopy to study the myelination of axons extending 

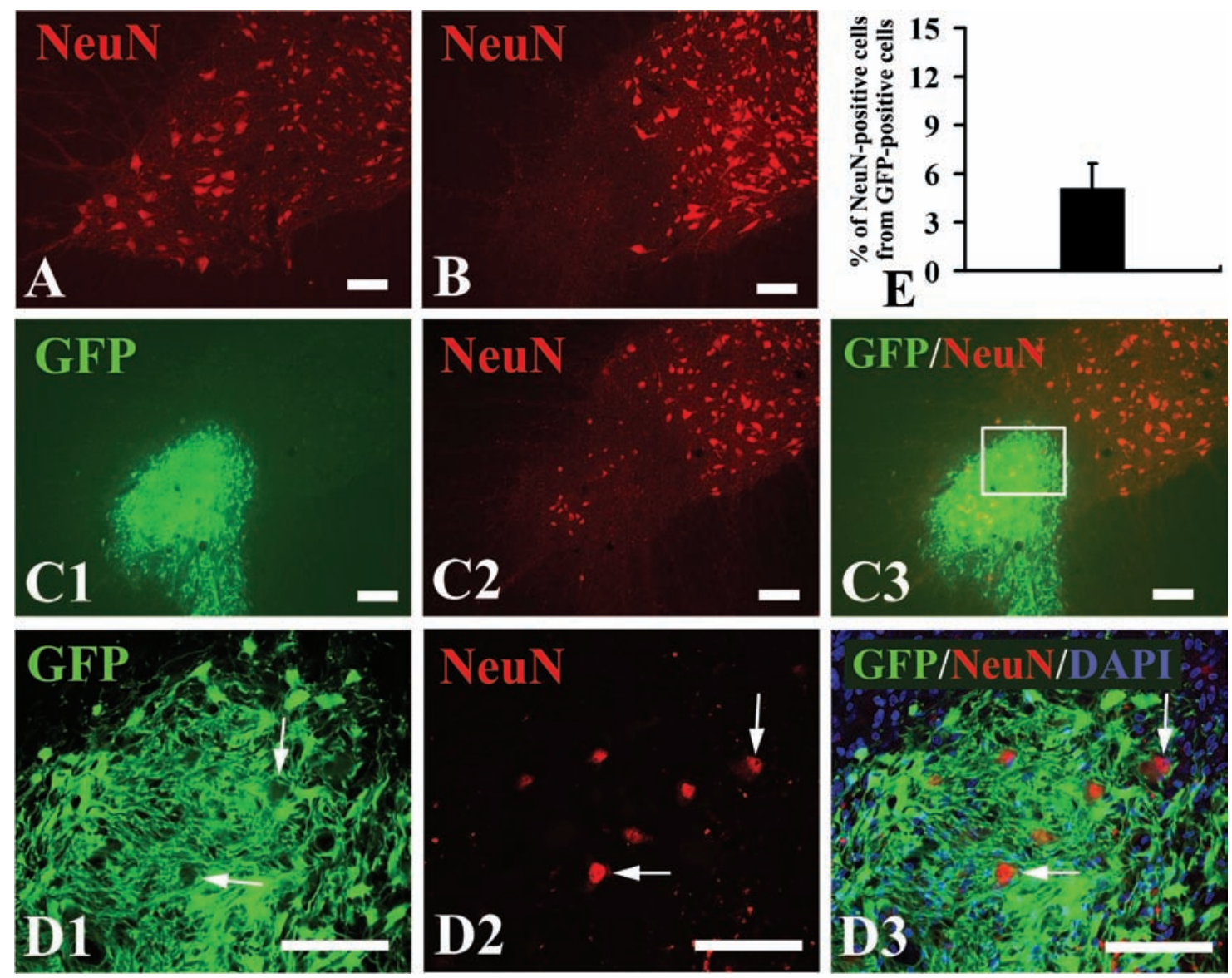

Figure 2. Neuronal differentiation of NPCs at 12 weeks after transplantation into the acutely avulsed ventral horn. (A) A cross section with NeuN staining shows the presence of motoneurons with large cell bodies in the normal ventral horn. (B) A cross section with NeuN staining shows the loss of motoneurons in the avulsed ventral horn. (C1-C3) A small proportion of grafted NPCs differentiated into NeuN-positive cells in the ventral horn. (D) Higher magnification of the outlined area in (C3). Some grafted cells with weaker intensity of GFP (arrow) in the ventral horn (D1) coexpressed NeuN (D2, D3). (E) Percentage of NeuN-positive neurons presented as mean \pm SEM. Scale bars: $50 \mu \mathrm{m}$.

from graft-derived neurons. Sections were incubated with mouse anti-NF200 (1:500; Chemicon) at $4^{\circ} \mathrm{C}$ overnight, followed by biotinylated anti-mouse IgG antibody (1:400; Chemicon). After being washed, sections were soaked for $1 \mathrm{~h}$ with an avidin-peroxidase complex (ABC Elite, Vector, Burlingame, CA) according to the manufacturer's suggestion and then reacted with diaminobenzidine for $5 \mathrm{~min}$. The reaction was stopped by washing extensively in PBS. After osmication in $1 \% \mathrm{OsO}_{4}$ for $1 \mathrm{~h}$, sections were washed again in $0.1 \mathrm{M}$ PB buffer, dehydrated through an ascending alcohol series ending in propylene oxide, and subsequently embedded using Eponate 12 Kit (Ted Pella, Redding, CA). Then, semithin sections were cut at $1 \mu \mathrm{m}$, mounted on glass slides, and stained with toluidine blue or Richardon's stain. Ultrathin sections were cut at $60 \mathrm{~nm}$ on a Reichert Ultracut S microtome (Leica); mounted on nickel grids, uranyl acetate, and lead citrate stained; and viewed under a Philips TEM/CM 10 electron microscope.

\section{Statistics Analysis}

Data were collected and expressed as mean \pm SEM. Student's two-tailed $t$ test was used for comparison of two experimental groups. Changes were identified as significant if $p<0.05$.

\section{RESULTS}

In Vitro Characterization of NPCs (Fig. 1)

To characterize P1 NPCs before transplantation, dissociated cells in single-cell suspension were plated onto coverslips coated with PLL/laminin (1:1 ratio) at a density of $10^{4} / \mathrm{cm}^{2}$ on a 24-well plate. With bFGF-supplemented culture medium, nearly all the cells showed a bipolar or multipolar morphology with small cell bodies and were immunoreactive for nestin, a marker for NPCs (Fig. 1A, J). MAP2 staining showed that less than $1 \%$ cells were positive $(0.96 \pm 0.1 \%$; Fig. 1B, J). Neither NF200-positive cells nor ChAT-positive cells were found in the culture 

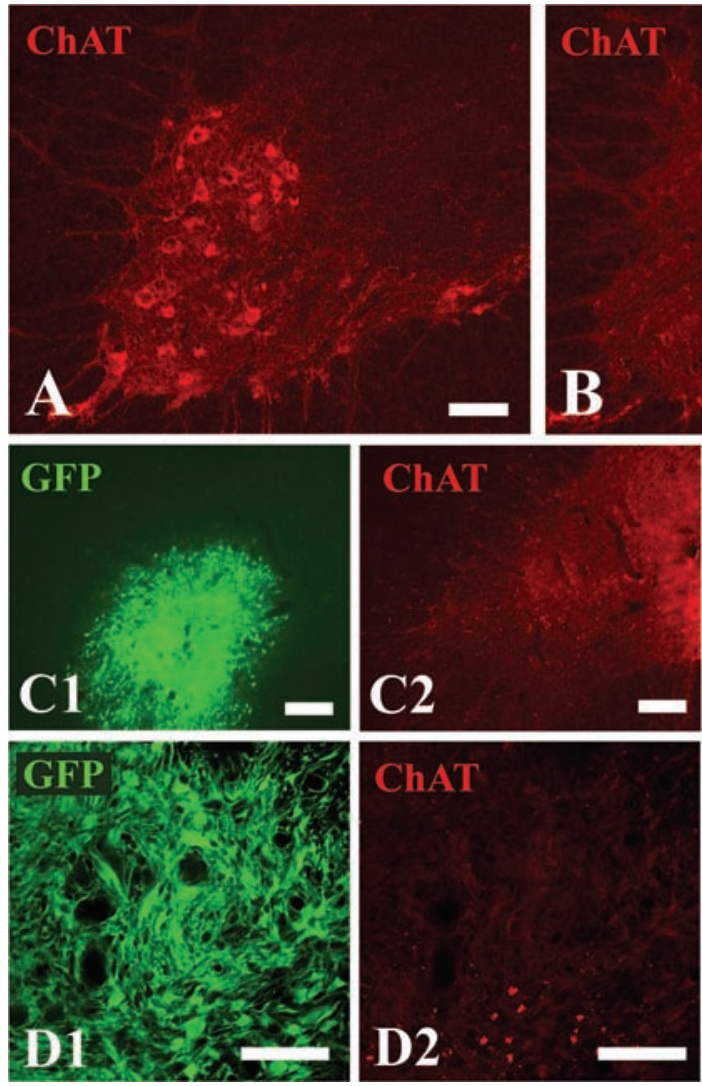

Figure 3. NPCs failed to differentiate into ChAT-positive neurons at 12 weeks after transplantation into the acutely avulsed ventral horn. (A) A cross section with ChAT staining showed the presence of ChAT-positive motoneurons in the normal ventral horn. (B) A cross section with ChAT staining showed complete loss of ChAT-positive neurons in the avulsed ventral horn. (C) A cross section with ChAT staining demonstrated that no graft-derived ChAT-positive cells were observed in the avulsed ventral horn. (D) Higher magnification of an area of interest in (C), further showing that no grafted cells colabeled with GFP and ChAT. Scale bars: $70 \mu \mathrm{m}$.

(Fig. 1C, D, J). NeuN staining showed that no cells were NeuN positive in the culture (Fig. 1E, J). These data confirmed that the cells remained at an immature stage. When bFGF was replaced with $1 \%$ fetal bovine serum (FBS), the NPCs began to differentiate. At the fifth day of culture with this differentiating medium, around $16.7 \%$ of the NPCs differentiated into MAP2-positive neurons (Fig. 1F, J). However, no NPCs were found to differentiate into ChAT-positive neurons in the in vitro differentiation system (Fig. 1G, J). The majority of NPCs differentiated into glial cells after 5 days in differentiating medium, with $73.5 \%$ differentiating into GFAP-positive astrocytes (Fig. 1H, J) and 8.4\% into Rip-positive oligodendrocytes (Fig. 1I, J).

\section{Grafted NPCs Failed to Generate Motoneuron-Like Cells in the Acutely Avulsed Ventral Horn}

Avulsion injury leads to massive motoneuron death in the ventral horn. NeuN staining demonstrated that, com- pared to the normal (Fig. 2A), massive neuronal loss was found in the avulsed ventral horn (Fig. 2B). To investigate whether grafted NPCs could respond to environmental cues to differentiate into motoneuron-like cells in the avulsed ventral horn, we performed immunohistochemical analyses at 12 weeks after transplantation. Transplanted NPCs were easily detected based on GFP expression. Cross sections of spinal cords from animals at 12 weeks after cell transplantation following root avulsion showed that the transplanted cells survived robustly in the avulsed ventral horn (Fig. 2C). NeuN staining revealed that only a small proportion of the grafted NPCs $(5.1 \pm 1.6 \%)$ differentiated into NeuN-positive cells, which were predominantly confined to the injection site in the ventral horn (Fig. 2C-E). These graft-derived neurons were much smaller in size compared to the host motoneurons (Fig. 2A). The intensity of GFP in NeuN-positive cells was much weaker than that in NeuN-negative cells (Fig. 2D). This seems to indicate that grafted NPCs 

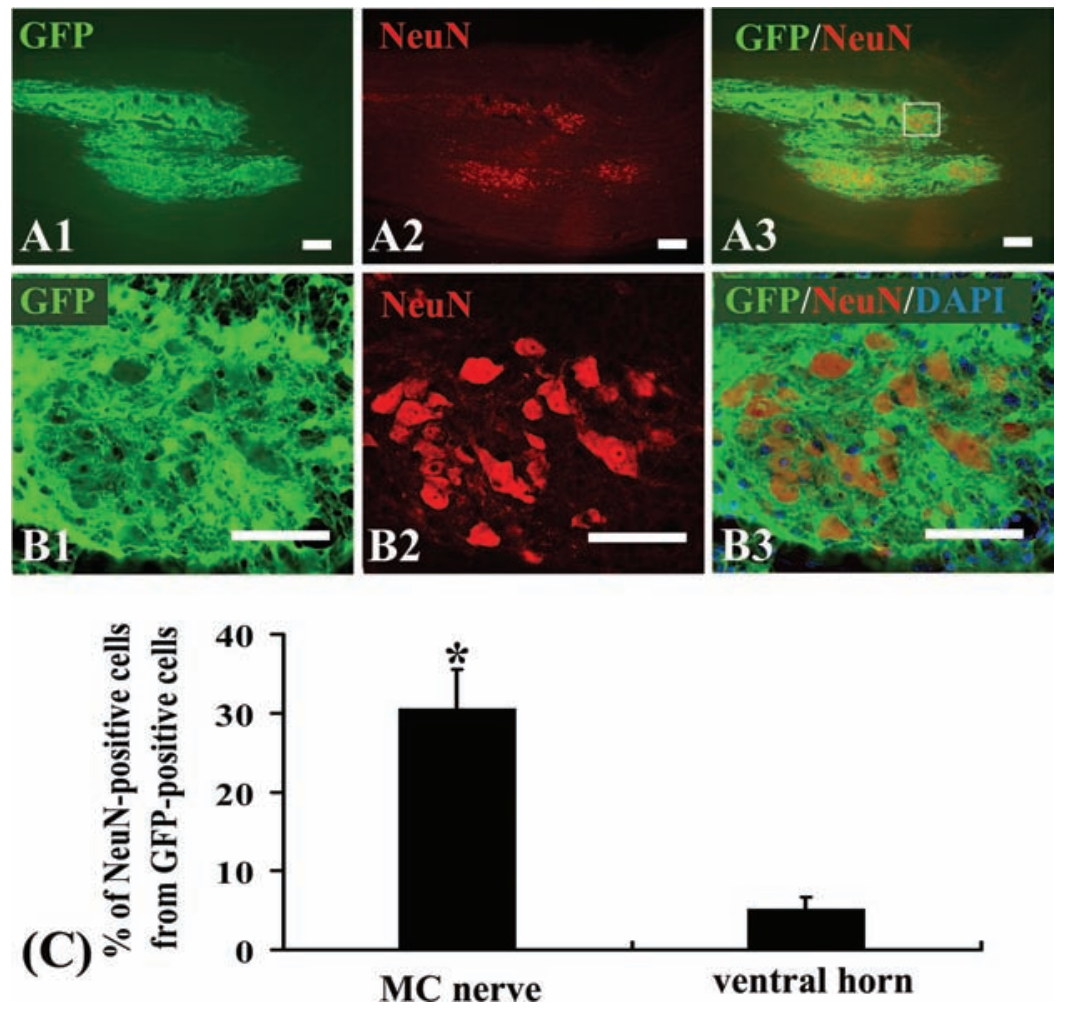

Figure 4. Neuronal differentiation of NPCs at 12 weeks after transplantation into the transected musculocutaneous (MC) nerve. (A) A longitudinal section of the nerve with NeuN staining shows that many grafted NPCs differentiated into NeuN-positive cells in the nerve. (B) Higher magnification of the outlined area in (A3). (C) Significantly more grafted NPCs differentiated into NeuN-positive neurons in the MC nerve than in the avulsed ventral horn. Data are presented as mean \pm SEM. ${ }^{*} p<0.001$ (Student's $t$ test). Scale bars: $35 \mu \mathrm{m}$.

down-regulated GFP expression after differentiation into neuron-like cells. ChAT is an enzyme that catalyzes acetylcholine synthesis for signal conduction through the neuromuscular junctions and is specifically expressed in motoneurons in the ventral horn. ChAT-positive motoneurons were present in the ventral horn of the normal side, showing typical motoneuron morphology with large cells bodies (Fig. 3A). Root avulsion led to a complete loss of host ChAT-positive cells in the injured ventral horn (Fig. 3B). Transplanted NPCs failed to generate ChATpositive neurons as demonstrated by the lack of positive staining for ChAT in the cell graft in the avulsed ventral horn (Fig. 3C, D). Similar to ChAT staining, NF200 staining did not detect any graft-derived NF200-positive cells in the ventral horn at 12 weeks after transplantation (data not shown).

\section{Grafted NPCs Generated Motoneuron-Like Cells in the Transected Musculocutaneous Nerve}

We then investigated the differentiation pattern of NPCs in the peripheral nerve. Immunostaining with a package of neuronal markers showed that the transected nerve is much more favorable for grafted NPCs to differentiate into neurons than the injured ventral horn of spinal cords. Substantial survival of GFP-expressing cells was observed in the musculocutaneous nerve at 12 weeks after transplantation (Fig. 4). Approximately 30.6 $\pm 3.5 \%$ NPCs differentiated into NeuN-positive cells in the musculocutaneous nerve at 12 weeks after transplantation, which is much higher than the $5.1 \pm 1.6 \%$ graft-derived NeuN-positive cells found in the ventral horn of the same animal (Fig. 4). To confirm the favorable effects of peripheral nerve on neuronal differentiation and to eliminate potential interactions between transplanted NPCs in the ventral horn and those in the peripheral nerve of the same animal, we transplanted NPCs into the single site of the animals (either the ventral horn or the transected musculocutaneous nerve only). NeuN staining revealed similar results of neuronal differentiation. Around $32.6 \pm 5.6 \%$ and $4.8 \pm 1.3 \%$ NPCs differentiated into NeuN-positive cells in the musculocutaneous nerve and injured ventral horn of independent animals, respectively (data not shown). In contrast to no graft-derived NF200-positive staining in the injured ventral horn (data not shown), nearly $18.7 \pm 3.1 \%$ NPCs were 

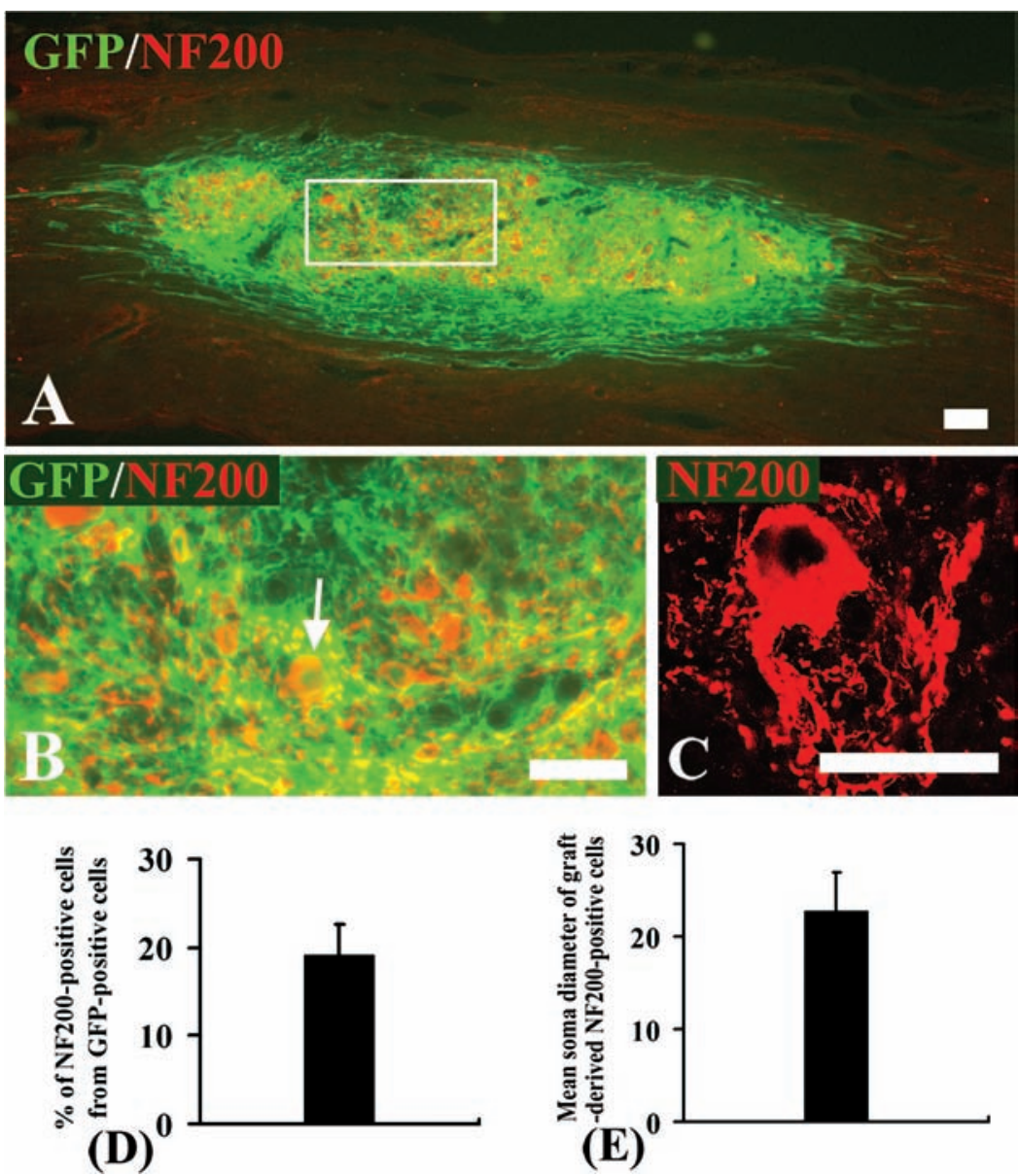

Figure 5. NPCs generated NF200-positive neurons at 12 weeks after transplantation into the transected musculocutaneous nerve. (A) A longitudinal section of the nerve with NF200 staining shows that many grafted NPCs differentiated into NF200-positive cells in the nerve. (B) Higher magnification of the outlined area in (A) showing graft-derived NF200-positive neurons with large cell bodies (arrow). (C) Under higher magnification, these cells exhibited extensive processes from their somatodendritic compartment. (D) Percentage of NF200-positive neurons presented as mean \pm SEM. (E) Mean soma diameter $( \pm$ SEM) of NF200-positive neurons. Scale bars: $75 \mu \mathrm{m}$.

detected to differentiate into NF200-positive cells in the nerve at 12 weeks after transplantation (Fig. 5A, B, D). Notably, these graft-derived NF200-positive neurons in the musculocutaneous nerve had enormously large cell bodies, with a mean soma diameter of $22.8 \pm 4.2 \mu \mathrm{m}$ (Fig. $5 \mathrm{~B}, \mathrm{C}, \mathrm{E}$ ). On detailed examination under higher magnification, these cells exhibited extensive processes from their somatodendritic compartment (Fig. 5C). ChAT staining further demonstrated that NPCs were able to give rise to ChATpositive neurons in the musculocutaneous nerve (Fig. 6A). Around $19.2 \pm 2.7 \%$ NPCs were found to be ChAT positive with large cell bodies (mean soma diameter, 17.6 $\pm 3.5 \mu \mathrm{m}$ ) at 12 weeks after transplantation (Fig. 6B, C).

We used a number of markers to examine the glial differentiation of grafted NPCs in the nerve. GFAP staining showed that the majority of NPCs differentiated into
GFAP-positive cells. Immunostaining with APC, MBP, or S-100 $\beta$ did not detect any positive staining in the cell graft, suggesting that NPCs failed to produce myelinating cells after transplantation into the nerve. We also investigated whether there were immature cells remaining in the graft at 12 weeks after transplantation into the injured musculocutaneous nerve. Nestin staining showed that no positive cells were detected in the graft (data not shown), suggesting that all transplanted cells had differentiated in the nerve. By 12 weeks after transection, we examined axonal extension of graft-derived motoneuron-like cells in the musculocutaneous nerve (Fig. 7A). The longitudinal section of the nerve from the vehicle control animals was devoid of any NF200 staining (Fig. 7B). However, NF200-labeled axons were present throughout the transected nerve from the cell- 

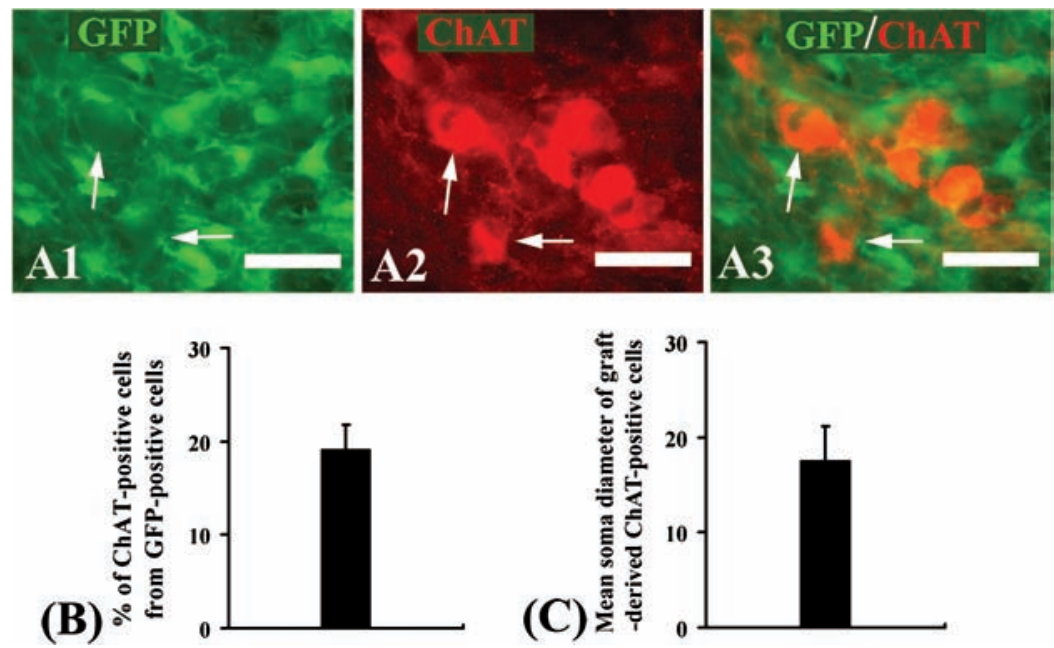

Figure 6. NPCs generated ChAT-positive neurons at 12 weeks after transplantation into the transected musculocutaneous nerve. (A) ChAT immunostaining of NPCs in the transected musculocutaneous nerve. Arrows indicate colabeling of ChAT and GFP. (B) Percentage of ChAT-positive neurons presented as mean \pm SEM. (C) Mean soma diameter $( \pm$ SEM) of ChAT-positive neurons. Scale bars: $40 \mu \mathrm{m}$.

implanted animals (Fig. 7C). Using anti-GFP antibody to intensify GFP signal, these NF200-positive axons were clearly shown to be colabeled with GFP, suggesting they were extending from the cell graft toward the nerve terminal (Fig. 7C). Immunoelectron microscopy showed the presence of Schwann cells in the nerve (Fig. 7D), and graft-derived axons were closely surrounded by Schwann cells, suggesting the beginning of myelination (Fig. 7E).

\section{Grafted NPCs Formed Functional Connections With Target Muscles and Reduced Denervated Muscle Atrophy}

A definitive character of functional motoneurons is that they have the capacity to functionally innervate the target muscle. The rat musculocutaneous nerve and biceps brachii model allows us to assess the functional innervation of grafted cells by histological studies and electrophysiological analyses. Muscle wet weight analysis showed that denervated biceps brachii muscle atrophied severely and its weight remained only one-fifth of the normal side at 12 weeks after transection of the musculocutaneous nerve (Fig. 8A, B). Transplantation of NPCs in the transected musculocutaneous nerve significantly reduced the target muscle atrophy, in that the biceps brachii muscle weight of the cell-implanted animals retained nearly $50 \%$ of the normal side (Fig. 8A, B). Sections of the biceps brachii muscle exhibited many neuromuscular junctions in cell-implanted animals as identified by rhodamine-conjugated $\alpha$-BT, and NF200positive axons were found to be in apposition to the neuromuscular junctions (Fig. 8C), whereas neither neuromuscular junctions nor NF200-positive axons were found in vehicle control animals (Fig. 8D). Figure 8E shows axon terminals attached to the neuromuscular junctions in the normal animals. When the peripheral nerve in isolated nerve-muscle preparations was stimulated, successful EMG response was easily induced in normal animals (Fig. 8F), while no EMG response was found in vehicle control animals (Fig. 8G). Notably, successful EMG response was also induced in all the animals with cell transplants (Fig. 8H1). To confirm that the muscle contractions resulted from neurotransmission by graft-derived motoneurons, the competitive nicotinic antagonist D-tubocurarine was applied to the recording solution. EMG activity was blocked with D-tubocurarine (Fig. 8H2), and this blockade was reversed after a 5-h washout period (Fig. 8H3). These data indicated functional innervation into the biceps brachii by implanted NPC-derived motoneurons.

\section{DISCUSSION}

Stem cell-based replacement strategies hold great promise in the treatment of motoneuron diseases due to their ability to replace lost, damaged, or dysfunctional neurons $(10,20)$. Using the rat brachial plexus injury model, we have demonstrated that spinal cord-derived NPCs, after transplantation in the transected musculocutaneous nerve, efficiently generate functional motoneurons as evidenced by typical morphology, specific molecular expression, and the capacity to innervate target muscles. This study suggests that in vitro expanded NPCs have therapeutic potential in cell therapy for motoneuron diseases due to their capability to give rise to functional motoneurons after transplantation in vivo. 

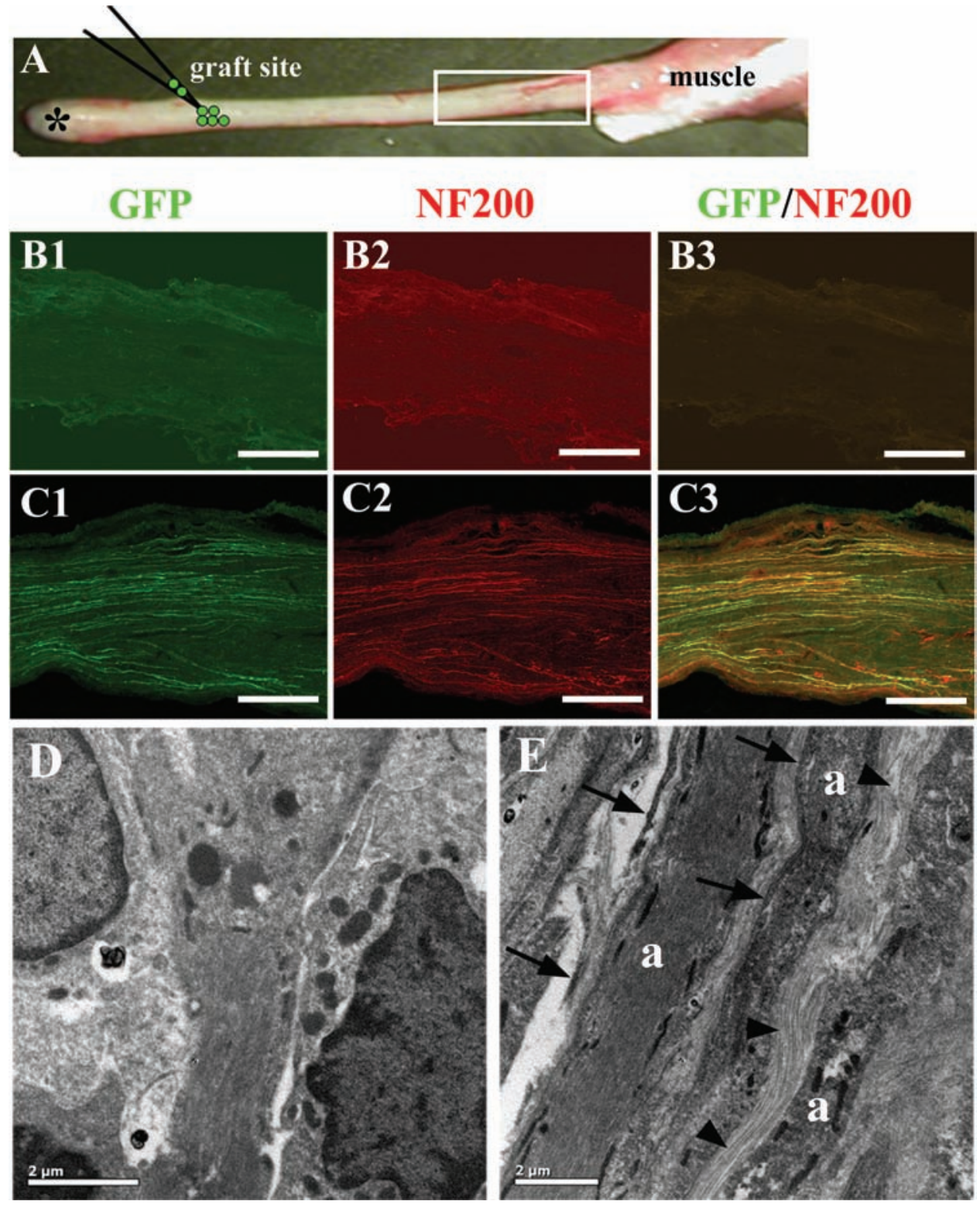

Figure 7. Graft-derived motoneuron-like cells extended axons along the nerve. (A) A schematic figure showing cell injection into the musculocutaneous nerve (*transected site; rectangle, an area used for studies of axonal extension as revealed in B, C). (B) The longitudinal section of the nerve in the vehicle control animals was devoid of any GFP or NF200 staining. (C) The longitudinal section of the nerve in the cell-implanted animals showed that NF200-labeled axons were present throughout the transected nerve. Using anti-GFP antibody to intensify GFP signal (C1), these NF200-positive axons were clearly shown to be colabeled with GFP (C2, C3). (D) An immunoelectron micrograph showing a motoneuron-like cell with two Schwann cell-like cells in the nerve. (E) An immunoelectron micrograph showing that graft-derived axons were closely surrounded by Schwann cells (a, axon; arrows, processes of Schwann cells; arrowheads, collagen fibers). Scale bars: $80 \mu \mathrm{m}(\mathrm{B}, \mathrm{C})$ and $2 \mu \mathrm{m}(\mathrm{D}, \mathrm{E})$.

It is still under consideration which stem cell type is the best source for treating specific neurological disorders such as motoneuron diseases. A number of studies have reported on the use of embryonic stem cells (ESCs) as a cell source of motoneurons. Both murine and human ESCs have been proven to be able to differentiate into functional motoneurons when exposed to Sonic Hedgehog (Shh) and retinoic acid (RA) $(17,36)$.
Spinally implanted ESC-derived motoneurons could survive in the ventral horn and extend axons into the ventral roots $(6,9)$. However, immune rejection and ethical controversy are major hurdles for the clinical application of ESCs. In addition, the difficulty in the purification of directed cells and the risk of teratoma formation are other major concerns related to the use of ESCs. 

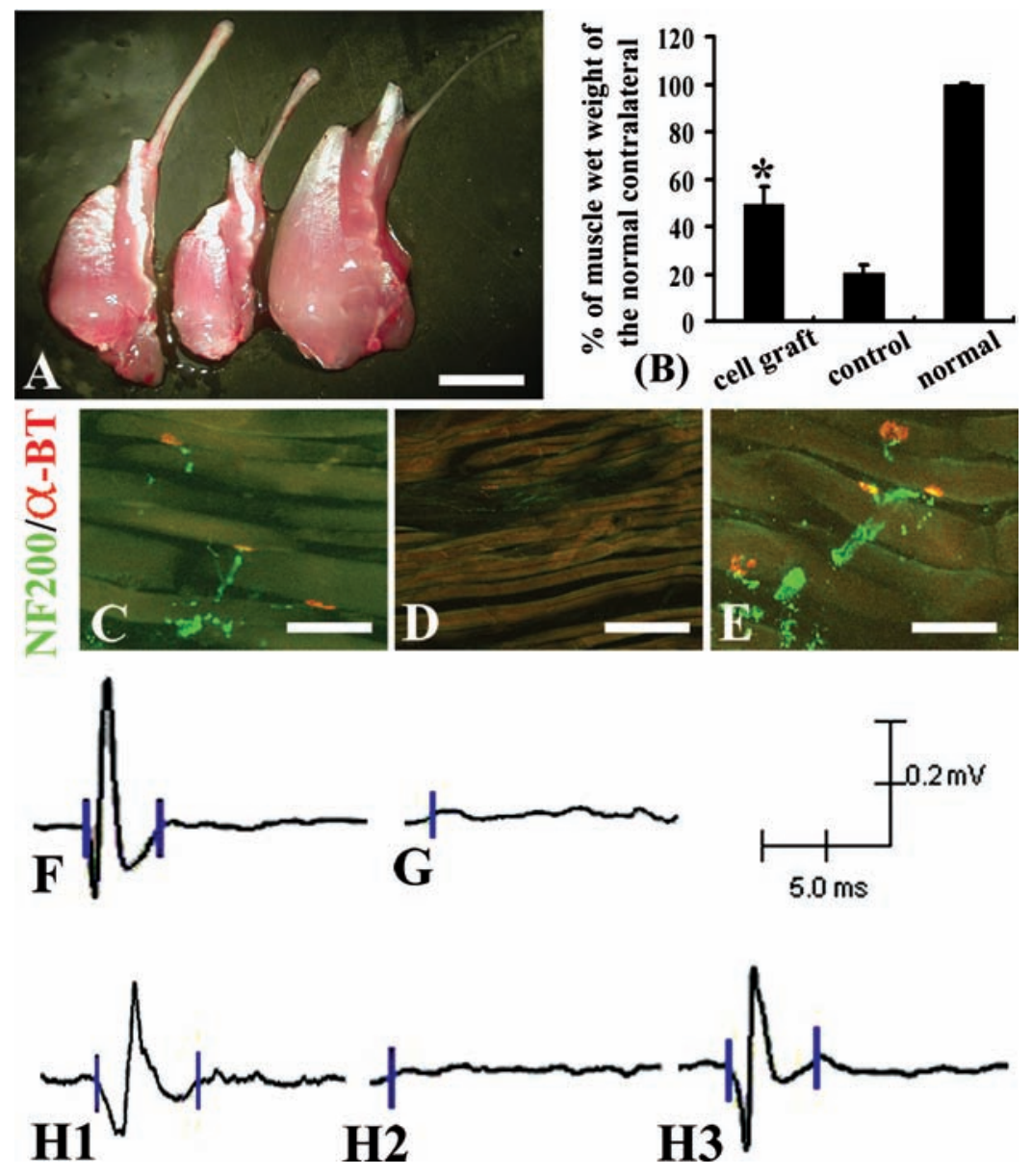

Figure 8. Grafted NPCs formed functional connections with the target muscle and reduced muscle atrophy. (A) Gross morphology of isolated musculocutaneous nerve and biceps brachii muscle at 12 weeks after cell or vehicle injection into the transected musculocutaneous nerve. Samples from an animal with cell graft (left), an animal with vehicle injection (middle), and a normal animal (right). (B) Transplantation of NPCs in the transected musculocutaneous nerve significantly reduced the target muscle atrophy compared to the vehicle control. ${ }^{*} p<0.001$ (Student's $t$ test). (C) A cross section of biceps brachii muscle showing that axon terminals labeled with NF200 (green) were in apposition to the neuromuscular junctions [labeled with rhodamine-conjugated $\alpha$-bungarotoxin $(\alpha-B$ T)] in the cell-grafted animals. (D) A cross section of biceps brachii muscle showing that neither neuromuscular junctions nor NF200-positive axons were found in the vehicle control animals. (E) A cross section of biceps brachii muscle showing axon terminals attached to the neuromuscular junctions in the normal animals. (F) Successful electromyographic (EMG) response was readily induced in the normal animals when the peripheral nerve in isolated nerve-muscle preparations was stimulated. $(G)$ No EMG response was found in vehicle control animals. (H) Successful EMG response was induced in animals with cell transplants (H1); EMG response was mediated through the neuromuscular junction, since application of D-tubocurarine effectively attenuated EMG activity (H2), which was recovered after a 5-h washout period (H3). Scale bars: $5 \mathrm{~mm}(\mathrm{~A})$ and $100 \mu \mathrm{m}(\mathrm{C}-\mathrm{E})$.

Induced pluripotent stem cells (iPSCs) have the advantage of eliminating immune rejection and ethical concerns because they are obtained from the host as well as have pluripotent behavior (30,37). Many studies have shown that iPSCs can successfully differentiate into functional motoneurons, opening an avenue for the clinical application of iPSC technology in the future $(2,7,12,16,26)$. However, there are a number of fundamental issues that need to be addressed on the use of iPSCs for neurological disorders, such as the low efficiency of neuronal differentiation with high variety across different iPS cell lines and the potential risks associated with transplantation of iPSC derivatives in vivo, such as carcinogenesis resulting either from overexpression of the oncogene or from insertional mutagenesis into the host genome.

NPCs are well recognized as a valuable source of cell transplant in the treatment of various neurological diseases because of their distinct attributes: they are committed to neural lineages and never give rise to inappropriate cell types; they are easily maintainable and readily engraftable; they have apparent abilities to integrate with 
the host environment and develop into integral cytoarchitectural components of CNS. Root avulsion of the brachial plexus leads to massive motoneuron degeneration and severe functional deficits (32). Transplantation of NPCs into the acutely avulsed ventral horn, however, failed to induce graft-derived motoneuron generation, suggesting that the environmental cues of the injured ventral horn are insufficient to induce NPCs to yield region-specific neurons for cell replacement. In contrast, when we transplanted NPCs into the transected musculocutaneous nerve, NPCs successfully differentiated into functional motoneurons to innervate the target muscle, indicating that the nerve holds sufficient cues to drive NPCs to yield motoneuron-like cells. Schwann cells in the nerve and molecular signals from terminal myocytes may contribute to the permissive milieu for NPC differentiation (33). The distinct differentiating fate of NPCs in the ventral horn and the peripheral nerve highlights the importance of host microenvironment affecting NPC differentiation. Very recently, we have reported that NPCs transplanted only at 2 weeks postavulsion were able to differentiate into ChAT-positive neurons, whereas transplantation immediately or at 6 weeks postavulsion did not induce NPCs to produce ChAT-positive neurons (28). The up-regulated expression levels of brain-derived neurotrophic factor (BDNF) and glial derived neurotrophic factor (GDNF) in the ventral horn at 2 weeks postavulsion are supposed to be the possible factor for rendering it as a supportive milieu for NPC differentiation. This study further indicates that, using NPCs to treat motoneuron diseases, it is necessary to modify the CNS microenvironment, which favors grafted cells to differentiate toward region-specific neurons.

The capacity of NPCs toward motoneuron differentiation has been explored by a number of studies $(4,5,15,18,21,23)$. However, all these studies did not investigate whether NPC-derived motoneurons could functionally innervate target muscles in vivo. Using the rat musculocutaneous nerve and biceps brachii model, we demonstrate that NPCs successfully generate functional motoneurons after transplantation in vivo. The model provides an excellent platform for us to evaluate the functional innervation of graft-derived motoneurons into the target muscle. Our study shows that in vitro expanded NPCs efficiently generate functional motoneurons, which extend axons to innervate the target muscle (biceps brachii) after transplantation into the transected musculocutaneous nerve and significantly reduce muscle atrophy.

In summary, the present study provides convincing evidence that NPCs have the potential to generate functional motoneurons in vivo, suggesting that NPCs could be a valuable cell source for the treatment of motoneuron diseases. The present study also highlights the importance of the future work to screen out the favorable molecules in the peripheral nerve and the inhibitory factors present in the ventral horn due to the distinct differentiating fate of NPCs in these two regions, which undoubtedly help us understand how to modify the host microenvironment to favor cell therapy.

ACKNOWLEDGMENTS: This study was supported by the Hong Kong Spinal Cord Injury Fund and the National Basic Research Program of China (973 Program, 2011CB504402). The authors declare no conflict of interest.

\section{REFERENCES}

1. Brustle, O.; Spiro, A. C.; Karram, K.; Choudhary, K.; Okabe, S.; McKay, R. D. In vitro-generated neural precursors participate in mammalian brain development. Proc. Natl. Acad. Sci. USA 94:14809-14814; 1997.

2. Cai. J.; Li, W.; Su, H.; Qin, D.; Yang, J.; Zhu, F.; Xu, J.; He, W.; Guo, X.; Labuda, K.; Peterbauer, A.; Wolbank, S.; Zhong, M.; Li, Z.; Wu, W.; So, K. F.; Redl, H.; Zeng, L.; Esteban, M. A.; Pei, D. Generation of human induced pluripotent stem cells from umbilical cord matrix and amniotic membrane mesenchymal cells. J. Biol. Chem. 285:1122711234; 2010.

3. Cao, Q. L.; Zhang, Y. P.; Howard, R. M.; Walters, W. M.; Tsoulfas, P.; Whittemore, S. R. Pluripotent stem cells engrafted into the normal or lesioned adult rat spinal cord are restricted to a glial lineage. Exp. Neurol. 167:48-51; 2001.

4. Corti, S.; Locatelli, F.; Papadimitriou, D.; Del Bo, R.; Nizzardo, M.; Nardini, M.; Donadoni, C.; Salani, S.; Fortunato, F.; Strazzer, S.; Bresolin, N.; Comi, G. P. Neural stem cells Lewis $\mathrm{X}^{+} \mathrm{CXCR} 4^{+}$modify disease progression in an amyotrophic lateral sclerosis model. Brain 130:1289$1305 ; 2007$.

5. Corti, S.; Nizzardo, M.; Nardini, M.; Donadoni, C.; Salani, S.; Ronchi, D.; Saladino, F.; Bordoni, A.; Fortunato, F.; Del Bo, R.; Papadimitriou, D.; Locatelli, F.; Menozzi, G.; Strazzer, S.; Bresolin, N.; Comi, G. P. Neural stem cell transplantation can ameliorate the phenotype of a mouse model of spinal muscular atrophy. J. Clin. Invest. 118:3316-3330; 2008.

6. Deshpande, D. M.; Kim, Y. S.; Martinez, T.; Carmen, J.; Dike, S.; Shats, I.; Rubin, L. L.; Drummond, J.; Krishnan, C.; Hoke, A.; Maragakis, N.; Shefner, J.; Rothstein, J. D.; Kerr, D. A. Recovery from paralysis in adult rats using embryonic stem cells. Ann. Neurol. 60:32-44; 2006.

7. Dimos, J. T.; Rodolfa, K. T.; Niakan, K.; Weisenthal, L.; Mitsumoto, H.; Chung, W.; Croft, G. F.; Saphier, G.; Leibel, R.; Goland, R.; Wichterle, H.; Henderson, C. E.; Eggan, $\mathrm{K}$. Induced pluripotent stem cells generated from patients with ALS can be differentiated into motor neurons. Science 321:1218-1221; 2008.

8. Dziewczapolski, G.; Lie, D. C.; Ray, J.; Gage, F. H.; Shults, C. W. Survival and differentiation of adult rat-derived neural progenitor cells transplanted to the striatum of hemiparkinsonian rats. Exp. Neurol. 183:653-664; 2003.

9. Harper, J. M.; Krishnan, C.; Darman, J. S.; Deshpande, D. M.; Peck, S.; Shats, I.; Backovic, S.; Rothstein, J. D.; Kerr, D. A. Axonal growth of embryonic stem cell-derived motoneurons in vitro and in motoneuron injured adult rats. Proc. Natl. Acad. Sci. USA 101:7123$7128 ; 2004$. 
10. Hedlund, E.; Hefferan, M. P.; Marsala, M.; Isacson, O. Cell therapy and stem cells in animal models of motor neuron disorders. Eur. J. Neurosci. 26:1721-1737; 2007.

11. Herrera, D. G.; Garcia-Verdugo, J. M.; Alvarez-Buylla, A. Adult-derived neural precursors transplanted into multiple regions in the adult brain. Ann. Neurol. 46:867-877; 1999.

12. Hu, B. Y.; Weick, J. P.; Yu, J.; Ma, L. X.; Zhang, X. Q.; Thomson, J. A.; Zhang, S. C. Neural differentiation of human induced pluripotent stem cells follows developmental principles but with variable potency. Proc. Natl. Acad. Sci. USA 107:4335-4340; 2010.

13. Isacson, O. The production and use of cells as therapeutic agents in neurodegenerative diseases. Lancet Neurol. $2: 417-424 ; 2003$.

14. Jessell, T. M. Neuronal specification in the spinal cord: Inductive signals and transcriptional codes. Nat. Rev. Genet. 1:20-29; 2000.

15. Kalyani, A. J.; Piper, D.; Mujtaba, T.; Lucero, M. T.; Rao, M. S. Spinal cord neuronal precursors generate multiple neuronal phenotypes in culture. J. Neurosci. 18:78567868; 1998

16. Karumbayaram, S.; Novitch, B. G.; Patterson, M.; Umbach, J. A.; Richter, L.; Lindgren, A.; Conway, A. E.; Clark, A. T.; Goldman, S. A.; Plath, K.; Wiedau-Pazos, M.; Kornblum, H. I.; Lowry, W. E. Directed differentiation of humaninduced pluripotent stem cells generates active motor neurons. Stem Cells 27:806-811; 2009.

17. Li, X. J.; Du, Z. W.; Zarnowska, E. D.; Pankratz, M.; Hansen, L. O.; Pearce, R. A.; Zhang, S. C. Specification of motoneurons from human embryonic stem cells. Nat. Biotechnol. 23:215-221; 2005.

18. Lin, S.; Xu, J.; Hu, S; Xu, L.; Zhang, C.; Wang, Y.; Gu, Y. Combined application of neutrophin-3 gene and neural stem cells is ameliorative to delay of denervated skeletal muscular atrophy after tibial nerve transection in rats. Cell Transplant. 20:381-390; 2011.

19. Lois, C.; Alvarez-Buylla, A. Proliferating subventricular zone cells in the adult mammalian forebrain can differentiate into neurons and glia. Proc. Natl. Acad. Sci. USA 90:2074-2077; 1993.

20. López-González, R.; Kunckles, P.; Velasco, I. Transient recovery in a rat model of familial amyotrophic lateral sclerosis after transplantation of motor neurons derived from mouse embryonic stem cells. Cell Transplant. 18:1171-1181; 2009.

21. MacDonald, S. C.; Fleetwood, I. G.; Hochman, S.; Dodd, J. G.; Cheng, G. K.; Jordan, L. M.; Brownstone, R. M. Functional motor neurons differentiating from mouse multipotent spinal cord precursor cells in culture and after transplantation into transected sciatic nerve. J. Neurosurg. 98:1094-1103; 2003.

22. Mayer-Proschel, M.; Kalyani, A. J.; Mujtaba, T.; Rao, M. S. Isolation of lineage-restricted neuronal precursors from multipotent neuroepithelial stem cells. Neuron 19:773-785; 1997.

23. Park, K. I.; Liu, S.; Flax, J. D.; Nissim, S.; Stieg, P. E.; Snyder, E. Y. Transplantation of .neural progenitor and stem cells: Developmental insights may suggest new therapies for spinal cord and other CNS dysfunction. J. Neurotrauma 16:675-687; 1999.

24. Reynolds, B. A.; Weiss, S. Generation of neurons and astrocytes from isolated cells of the adult mammalian central nervous system. Science 255:1707-1710; 1992.

25. Snyder, E. Y.; Deitcher, D. L.; Walsh, C.; Arnold-Aldea, S.; Hartwieg, E.; Cepko, C. L. Multipotent neural cell lines can engraft and participate in development of mouse cerebellum. Cell 68:33-51; 1992.

26. Soldner, F.; Hockemeyer, D.; Beard, C.; Gao, Q.; Bell, G.; Cook, E. G.; Hargus, G.; Blak, A.; Cooper, O.; Mitalipova, M.; Isacson, O.; Jaenisch, R. Parkinson's disease patientderived induced pluripotent stem cells free of viral reprogramming factors. Cell 136:964-977; 2009.

27. Su, H.; Chu, T. H; Wu, W. Lithium enhances proliferation and neuronal differentiation of neural progenitor cells in vitro and after transplantation into the adult rat spinal cord. Exp. Neurol. 206:296-307; 2007.

28. Su, H.; Wu, Y.; Yuan, Q.; Guo, J.; Zhang, W.; Wu, W. Optimal time point for neuronal generation of transplanted neural progenitor cells in injured spinal cord following root avulsion. Cell Transplant. 20:167-176; 2011.

29. Su, H.; Zhang, W.; Guo, J.; Guo, A.; Yuan, Q.; Wu, W. Neural progenitor cells enhance the survival and axonal regeneration of injured motoneurons after transplantation into the avulsed ventral horn of adult rats. J. Neurotrauma 26:67-80; 2009.

30. Takahashi, K.; Tanabe, K.; Ohnuki, M.; Narita, M.; Ichisaka, T.; Tomoda, K.; Yamanaka, S. Induction of pluripotent stem cells from adult human fibroblasts by defined factors. Cell 131:861-872; 2007.

31. Temple, S. The development of neural stem cells. Nature 414:112-117; 2001.

32. Terzis, J. K.; Vekris, M. D.; Soucacos, P. N. Outcomes of brachial plexus reconstruction in 204 patients with devastating paralysis. Plast. Reconstr. Surg. 104:1221$1240 ; 1999$

33. Thomas, C. K.; Erb, D. E.; Grumbles, R. M.; Bunge, R. P. Embryonic cord transplants in peripheral nerve restore skeletal muscle function. J. Neurophysiol. 84:591-595; 2000.

34. Uchida, N.; Buck, D. W.; He, D.; Reitsma, M. J.; Masek, M.; Phan, T.; Tsukamoto, A. S.; Gage, F. H.; Weissman, I. L. Direct isolation of human central nervous system stem cells. Proc. Natl. Acad. Sci. USA 97:14720-14725; 2000.

35. Wang, G.; Ao, Q.; Gong, K.; Zuo, H.; Gong, Y.; Zhang, X. Synergistic effect of neural stem cells and olfactory ensheathing cells on repair of adult rat spinal cord injury. Cell Transplant. 19:1325-1337; 2010.

36. Wichterle, H.; Lieberam, I.; Porter, J. A.; Jessell, T. M. Directed differentiation of embryonic stem cells into motor neurons. Cell 110:385-397; 2002.

37. Yu, J.; Vodyanik, M. A.; Smuga-Otto, K.; AntosiewiczBourget, J.; Frane, J. L.; Tian, S.; Nie, J.; Jonsdottir, G. A.; Ruotti, V.; Stewart, R.; Slukvin, I. I.; Thomson, J. A. Induced pluripotent stem cell lines derived from human somatic cells. Science 318:1917-1920; 2007. 